\title{
Rapid convergence of the Weinberg expansion of the deuteron stripping amplitude
}

\author{
D. Y. Pang, ${ }^{1}$ N. K. Timofeyuk, ${ }^{2}$ R. C. Johnson, ${ }^{2}$ and J. A. Tostevin ${ }^{2}$ \\ ${ }^{1}$ School of Physics and Nuclear Energy Engineering, Beihang University, Beijing 100191, China \\ ${ }^{2}$ Department of Physics, Faculty of Engineering and Physical Sciences, University of Surrey, Guildford, Surrey GU2 7XH, United Kingdom
}

(Received 1 May 2013; published 26 June 2013)

\begin{abstract}
Theories of $(d, p)$ reactions frequently use a formalism based on a transition amplitude that is dominated by the components of the total three-body scattering wave function where the spatial separation between the incoming neutron and proton is confined by the range of the $n-p$ interaction, $V_{n p}$. By comparison with calculations based on the continuum discretized coupled channels method we show that the $(d, p)$ transition amplitude is dominated by the first term of the expansion of the three-body wave function in a complete set of Weinberg states. We use the ${ }^{132} \mathrm{Sn}(d, p){ }^{133} \mathrm{Sn}$ reaction at 30 and $100 \mathrm{MeV}$ as examples of contemporary interest. The generality of this observed dominance and its implications for future theoretical developments are discussed.
\end{abstract}

DOI: 10.1103/PhysRevC.87.064613

PACS number(s): 24.50.+g, 25.45.Hi, 25.60.Je

\section{INTRODUCTION}

There is growing interest and activity in transfer reaction studies using radioactive beams, driven by increased secondary beam intensities and motivated by the search for new physics at the edge of nuclear stability [1,2] and by the need for low-energy reaction rates for astrophysical applications [3,4]. The $(d, p)$ reaction, measured in inverse kinematics, is well suited for these purposes. It can provide spin-parity assignments for nuclear states, allow determination of spectroscopic strengths of single-particle configurations, and give asymptotic normalization coefficients in the tail of overlap functions. The reliability of this deduced nuclear structure information depends on the existence of a reaction theory that describes adequately the mechanism of the $(d, p)$ reaction.

This paper uses a formulation of the $A(d, p) B$ reaction amplitude that emphasizes the components of the total neutron + proton + target scattering wave function where the spatial separations between the incoming neutron and proton are confined by the range of the $n-p$ interaction, $V_{n p}$. These components contain both the bound and continuum states of the $n$ - $p$ system. Since the $n-p$ binding energy in the deuteron is small and the optical potentials that generate the tidal breakup forces are smooth functions of position, the strength of inelastic excitations to the $n-p$ continuum is expected to be concentrated at low $n-p$ relative energies. This suggests that the coupling effects between different $n-p$ states can be treated adiabatically and leads to a simple prescription for calculating the scattering wave function at small $n-p$ separations [5].

In the adiabatic model the $A(d, p) B$ transition amplitude has exactly the same structure as that of the distorted-wave Born approximation (DWBA), for which many computer codes are available, which has led to its widespread use [2,5-9]. The adiabatic model frequently provides significant improvements over the DWBA for $A(d, p) B$ angular distributions and giving consistent results for nuclear structure information [9].

There are two key ingredients in the adiabatic model: (i) the assumption that only components of the three-body scattering wave function with small $n-p$ separation are needed for the $A(d, p) B$ transition amplitude and (ii) the validity of the adiabatic treatment of deuteron breakup at the nuclear surface.
The primary purpose of this paper is to show that assumption (i) is justified for a useful range of reaction energies when it is implemented in terms of a precisely defined projection of the three-body scattering wave function. This projection will be shown to involve the first Weinberg state component of the full wave function.

Investigations of how assumption (ii) influences the predicted $(d, p)$ cross sections were carried out using the quasiadiabatic model $[10,11]$, the Weinberg states expansion (WSE) method $[8,12]$, the continuum discretized coupled channels (CDCC) method, and also Faddeev equation methods [13,14]. The importance of nonadiabatic effects has been found to depend on the target and incident energy and, in the worst cases, these affected both the shapes and the magnitudes of the calculated differential cross sections [12,14]. There is therefore an important need to provide a practical way of introducing corrections to the adiabatic approximation. Our aim here is to provide a suitable definition of the projection of the full scattering wave function implied by assumption (i), which we call the first Weinberg projection. We show that this projection, which is a function of only a single vector coordinate, dominates the calculation of the $A(d, p) B$ transition amplitude. This result implies that to include effects beyond the adiabatic approximation one can focus on improvements to the calculation of this projection only.

The CDCC method for solving the three-body problem does not use the adiabatic approximation (ii). From a practical point of view it is well adapted to the study of deuteron breakup effects on $A(d, p) B$ reactions. In principle in the CDCC method one attempts to calculate the three-body scattering wave function in the whole six-dimensional coordinate space of the neutron + proton $+\operatorname{target}(n+p+A)$ three-body system. Our approach is to compare calculations of the $(d, p)$ transition amplitude made using a complete CDCC wave function with calculations which retain only the first few Weinberg components of the full CDCC wave function.

In Sec. II we describe how the projection procedure mentioned above is related to the Weinberg state and CDCC expansion methods and we connect these. In Sec. III we 
construct the Weinberg components using the CDCC wave functions and in Sec. IV we compare calculations of the $(d, p)$ transition amplitudes using the first few Weinberg components. We summarize our results in Sec. V.

\section{THREE-BODY WAVE FUNCTION AND ITS EXPANSION IN THE CDCC AND WEINBERG STATE BASES}

In the absence of inelastic excitations of the target and residual nuclei $A$ and $B$ in the incident and outgoing channels, the transition amplitude of the $A(d, p) B$ reaction can be written as [5]

$$
T_{d p}=\left\langle\chi_{p}^{(-)} I_{A B}\left|V_{n p}\right| \Psi^{(+)}\right\rangle
$$

Here $\chi_{p}^{(-)}$is the outgoing proton distorted wave (where we neglect certain $1 / A$ corrections [15]), $I_{A B}$ is the overlap function between the wave functions of $A$ and $B, V_{n p}$ is the neutron-proton interaction, and $\Psi^{(+)}$is the projection of the full many-body wave function onto the three-body, $n+p+A$, channel with $A$ in its ground state. The effect of coupling to excited states of $A$ is implicitly taken into account through the use of complex nucleon optical potentials, but contributions from transitions that explicitly excite components of $A$ in the initial state and $B$ in the final state are ignored. We assume that $\Psi^{(+)}$satisfies the Schrödinger equation

$$
\begin{aligned}
& {\left[E_{d}+i \epsilon-H_{n p}-T_{R}-U_{n}\left(\boldsymbol{r}_{n}\right)-U_{p}\left(\boldsymbol{r}_{p}\right)\right] \Psi^{(+)}(\boldsymbol{r}, \boldsymbol{R})} \\
& \quad=i \epsilon \phi_{d}(\boldsymbol{r}) e^{i \boldsymbol{K}_{d} \cdot \boldsymbol{R}},
\end{aligned}
$$

where $H_{n p}=T_{r}+V_{n p}$ is the $n$ - $p$ relative motion Hamiltonian. Here $E_{d}=E_{\text {c.m. }}-\epsilon_{d}$, where $\epsilon_{d}$ is the deuteron binding energy and $E_{\mathrm{c} . \mathrm{m} .}$ is the three-body energy in the center-of-mass system. $U_{n}$ and $U_{p}$ are the optical model potentials for the neutron and the proton with the target nucleus, respectively, and $\boldsymbol{K}_{d}$ is the wave number associated with $E_{d}$. The coordinates $\boldsymbol{r}_{p}$ and $\boldsymbol{r}_{n}$ are the proton and neutron coordinates with respect to the target $A$ while $\boldsymbol{r}=\boldsymbol{r}_{p}-\boldsymbol{r}_{n}$ and $\boldsymbol{R}=\frac{1}{2}\left(\boldsymbol{r}_{n}+\boldsymbol{r}_{p}\right)$ are the relative and c.m. coordinates of the $n-p$ pair. Also,

$$
T_{r}=-\frac{\hbar^{2}}{2 \mu_{n p}} \nabla_{r}^{2} \quad \text { and } \quad T_{R}=-\frac{\hbar^{2}}{2 \mu_{d A}} \nabla_{R}^{2}
$$

are the kinetic energy operators associated with $\boldsymbol{r}$ and $\boldsymbol{R}$, with $\mu_{n p}$ and $\mu_{d A}$ the reduced masses of the $n-p$ pair and the $n+$ $p+A$ system, respectively. The right-hand side of Eq. (2) specifies the incident boundary condition of a deuteron with initial wave function $\phi_{d}$ and the physical total wave function is to be calculated in the limit $\epsilon \rightarrow 0+$. The superscripts on $\chi_{p}^{(-)}$and $\Psi^{(+)}$indicate that they obey ingoing and outgoing waves boundary conditions, respectively. For simplicity, these superscripts are omitted in the following text.

In the next two sections we describe two expansion schemes for the total wave function $\Psi(\boldsymbol{r}, \boldsymbol{R})$.

\section{A. The Weinberg states expansion}

For $n-p$ separations $r$ within the range of $V_{n p}$, the wave function $\Psi(\boldsymbol{r}, \boldsymbol{R})$ has the expansion $[8,12]$

$$
\Psi(\boldsymbol{r}, \boldsymbol{R})=\sum_{i} \phi_{i}^{W}(\boldsymbol{r}) \chi_{i}^{W}(\boldsymbol{R}),
$$

where the Weinberg states, $\phi_{i}^{W}$, are solutions of the equation

$$
\left[-\epsilon_{d}-T_{r}-\alpha_{i} V_{n p}\right] \phi_{i}^{W}(\boldsymbol{r})=0, \quad i=1,2, \ldots
$$

with fixed energy $-\epsilon_{d}$ and eigenvalues $\alpha_{i}$. For radii $r>r_{i}$, where $r_{i}$ is such that $\alpha_{i} V_{n p}(r)$ is negligible, all of the Weinberg states decay exponentially, like the deuteron ground-state wave function. For $r<r_{i}$, they oscillate with a wavelength that varies with $i$, becoming increasingly oscillatory with increasing $i$ (see the examples given in [12] for the case of a Hulthén form for $V_{n p}$ ).

The Weinberg states form a complete set of functions of $r$ for regions of the $r$ axis on which $V_{n p}$ is nonvanishing. They are therefore well adapted to expanding $\Psi$ in this region. They do not satisfy the usual orthonormality relation but instead satisfy

$$
\left\langle\phi_{i}^{W}\left|V_{n p}\right| \phi_{j}^{W}\right\rangle=-\delta_{i j},
$$

where the value -1 for $i=j$ has been chosen for convenience.

This form of orthonormality, with a weight factor $V_{n p}$, means that if one wishes to represent an arbitrary state $\varphi(r)$ as a linear superposition of Weinberg states then the unique choice of coefficients $a_{i}$ which minimizes the difference

$$
\Delta=\int d \boldsymbol{r} V_{n p}\left|\varphi-\sum_{i} a_{i} \phi_{i}^{W}\right|^{2}
$$

is

$$
a_{i}=-\left\langle\phi_{i}^{W}\left|V_{n p}\right| \varphi\right\rangle .
$$

Use of a factor $V_{n p}$ in Eq. (6), which weights $r$ values according to $V_{n p}$, provides a natural scheme for constructing the expansion coefficients for states of $n$ - $p$ relative motion for use in the $(d, p)$ transition amplitude.

\section{B. The CDCC basis method}

The CDCC method involves the expansion of $\Psi(\boldsymbol{r}, \boldsymbol{R})$ in terms of a complete set of $n$ - $p$ continuum bin states $\phi_{i}^{\text {bin }}$ (see, e.g., Ref. [16]), written

$$
\Psi(\boldsymbol{r}, \boldsymbol{R})=\phi_{d}(\boldsymbol{r}) \chi_{0}(\boldsymbol{R})+\sum_{i=1} \phi_{i}^{\mathrm{bin}}(\boldsymbol{r}) \chi_{i}^{\mathrm{bin}}(\boldsymbol{R}) .
$$

The bin states are linear superpositions of continuum eigenfunctions of $H_{n p}$, on chosen intervals $\Delta k_{i}$ of $n-p$ continuum wave numbers, and are orthogonal in the usual sense. So, the projection of the three-body Schrödinger equation of Eq. (2) onto this set of spatially extended bin states leads to a set of coupled-channel equations for the channel wave functions $\chi_{i}^{\text {bin }}(\boldsymbol{R})$. The coupling potentials, generated from the nucleon optical potentials, are long-ranged and link parts of the wave function from all $n-p, n-A$, and $p$ - $A$ separations.

These CDCC equations can be solved numerically and their convergence properties have been intensively studied. 


\section{Connection between the CDCC and Weinberg basis wave functions}

It is known from experience with CDCC calculations that the energy range of $n-p$ continuum states that are coupled to the incident deuteron channel is limited to tens of $\mathrm{MeV}$. Thus, we expect that inside the range of $V_{n p}$ the wave function $\Psi(\boldsymbol{r}, \boldsymbol{R})$ will not be a strongly oscillatory function of $r$ and only a few terms of the Weinberg expansion will be needed to evaluate the $(d, p)$ matrix element. Note that this has nothing to do with the strength of the coupling between Weinberg components in $\Psi(\boldsymbol{r}, \boldsymbol{R})$ or how rapidly the Weinberg expansion for $\Psi(\boldsymbol{r}, \boldsymbol{R})$ itself converges, but rather it relates to how rapid the convergence of the sequence of contributions to the $(d, p)$ amplitude is from the different Weinberg components. We do not obtain the latter from a set of coupled equations, as, e.g., was done successfully in Ref. [12], but rather from the CDCC expansion of $\Psi(\boldsymbol{r}, \boldsymbol{R})$. The quantitative issues arising from a comparison with the approach of Ref. [12] will be addressed elsewhere.

To connect the Weinberg and CDCC components of $\Psi(\boldsymbol{r}, \boldsymbol{R})$ we project $\Psi$, expressed in the CDCC basis, onto individual Weinberg states using the orthogonality property of Eq. (5). The Weinberg distorted waves, $\chi_{i}^{W}$, and those of the CDCC basis, $\chi_{j}^{\text {bin }}$, are related using

$$
\chi_{i}^{W}(\boldsymbol{R})=C_{i 0} \chi_{0}(\boldsymbol{R})+\sum_{j=1} C_{i j} \chi_{j}^{\mathrm{bin}}(\boldsymbol{R}) .
$$

The transformation coefficients $C_{i j}$ are given by

$$
\begin{aligned}
& C_{i 0}=-\left\langle\phi_{i}^{W}\left|V_{n p}\right| \phi_{d}\right\rangle \quad(=0, i \neq 1), \\
& C_{i j}=-\left\langle\phi_{i}^{W}\left|V_{n p}\right| \phi_{j}^{\text {bin }}\right\rangle \quad(i, j=1,2, \ldots) .
\end{aligned}
$$

These coefficients also appear in the formulas

$$
\left|\phi_{j}^{\mathrm{bin}}\right\rangle=\sum_{i} C_{i j}\left|\phi_{i}^{W}\right\rangle
$$

and

$$
\int d \boldsymbol{r} V_{n p}\left|\phi_{j}^{\mathrm{bin}}(r)\right|^{2}=\sum_{i}\left|C_{i j}\right|^{2}
$$

that quantify the contribution of each Weinberg state to a particular CDCC bin state, in the presence of the weight factor $V_{n p}$.

These $C_{i j}$ are determined entirely by the bound and scattering states of $V_{n p}$ in the energy range of the relevant bin states. They do not depend on any other details of the reaction, such as the deuteron incident energy, the transferred angular momentum, or the structure of the target nuclei. The values of $C_{i j}$ do depend on how the CDCC bin states were constructed, the bin sizes $\Delta k_{i}$, etc.; however, we have checked that the changes in the computed $\chi_{i}^{W}$ are less than $0.1 \%$ with typical choices of bin sizes, such as $\Delta k_{i} \approx 0.1-0.15 \mathrm{fm}^{-1}$. Throughout this work a Hulthén potential was used for $V_{n p}$, namely,

$$
V_{n p}(r)=V_{0} /\left(e^{\beta r}-1\right),
$$

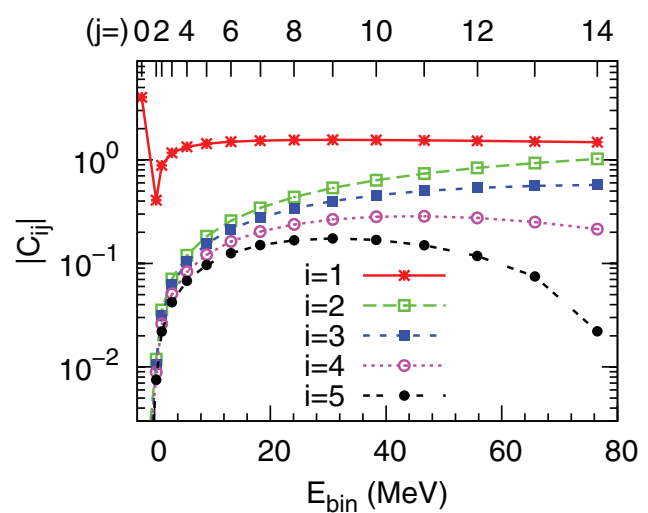

FIG. 1. (Color online) CDCC bin state to Weinberg state transformation coefficients $C_{i j}$, of Eq. (9), for Weinberg states $i=1,2, \ldots, 5$ and CDCC bin states $j=1,2, \ldots, 14$. The deuteron ground state is denoted by $j=0$. The CDCC bins were calculated up to $n$ - $p$ relative momenta $k_{\max }=1.4 \mathrm{fm}^{-1}$ in steps $\Delta k_{i}=0.1 \mathrm{fm}^{-1}$. See Sec. III for full details.

with parameters $V_{0}=-84.86 \mathrm{MeV}$ and $\beta=1.22 \mathrm{fm}^{-1}$ [12]. Only $s$-wave continuum states were included. These give the largest contribution to $\Psi(\boldsymbol{r}, \boldsymbol{R})$ at small $r$.

In Fig. 1 we show the calculated $C_{i j}$ for $i \leqslant 5$ for bin states $\phi_{j}^{\text {bin }}$ calculated from CDCC calculations using the computer code FRESCO [17]. The lower and upper horizontal axes show the $n-p$ continuum energies included in the CDCC and the label of the different bins, with $j=1, \ldots, 14$, respectively. The point with $j=0$ shows the $C_{10}$ that connects with the deuteron ground state. Each line then corresponds to a different Weinberg state, $\phi_{i}^{W}$.

For the $(d, p)$ reaction the most relevant continuum energies lie in the range 0 to $40 \mathrm{MeV}$. From Eq. (11) and the $i$ dependence of the $C_{i j}$ for the lower energy (and $j$ ) bins in Fig. 1, we see that the bin states in the relevant energy range are dominated by the first Weinberg component with only small contributions from Weinberg states $i=2-5$. This dominance is particularly marked for the low-energy continuum, which is the most strongly coupled to the deuteron ground state by the breakup mechanism and that has the largest $\chi_{i}^{\text {bin }}(\boldsymbol{R})$ in Eq. (8). At the higher continuum energies the bin states are mixtures of several Weinberg states, as was expected.

In Eq. (9), this dominance of the $i=1$ coefficients for low continuum energies will make $\chi_{1}^{W}$ the dominant Weinberg distorted wave provided the contributions from continuum bins with energies greater than of order $30 \mathrm{MeV}$ are not large. In the next section we present the details of CDCC calculations and show that these qualitative observations are borne out quantitatively for typical $(d, p)$ reactions and energies.

\section{CONSTRUCTION OF THE $\chi_{i}^{W}$ FROM THE CDCC WAVE FUNCTION}

In this section, as relevant topical examples, we construct the Weinberg distorted waves $\chi_{i}^{W}$ for the ${ }^{132} \mathrm{Sn}(d, p)^{133} \mathrm{Sn}$ reaction at deuteron incident energies $E_{d}=100$ and $30 \mathrm{MeV}$ at which the contributions from closed channels are negligible. Neutron-rich target nuclei, for inverse kinematics $(d, p)$ 
experiments at such energies per nucleon, are available at several modern radioactive ion beam facilities such as RIKEN [18], GANIL [19], NSCL [20], FLNR at Dubna [21], and IMP at Lanzhou [22].

We solved the CDCC equations using nucleon optical potentials, $U_{n}$ and $U_{p}$, evaluated at half the incident deuteron energy, taken from the KD02 systematics [23]. Only the central parts of these potentials were used. Both the nuclear and Coulomb potentials were used in constructing the coupling potentials. The continuum bin states $\phi^{\text {bin }}$ were computed by discretizing the $s$-wave $n-p$ continuum using $\Delta k_{i}$ of 0.1 and $0.05 \mathrm{fm}^{-1}$ up to $k_{\max }=1.4$ and $0.75 \mathrm{fm}^{-1}$, corresponding to maximum continuum energies of 81.9 and $23.5 \mathrm{MeV}$, for $E_{d}=100$ and $30 \mathrm{MeV}$, respectively. The coupled-channels CDCC equations were solved up to $R_{\max }=100 \mathrm{fm}$ because of the long-range nature of the CDCC couplings [24]. The CDCC calculations were performed using the computer code FRESCO [17].

The $\chi_{i}^{W}$ were constructed from Eq. (8) using the coefficients $C_{i j}$ discussed in the previous section. It was found that bins up to a maximum continuum energy of $25 \mathrm{MeV}$ are sufficient for the convergence of $\chi_{1}^{W}$ for both deuteron incident energies. This is illustrated in Figs. 2(a) and 2(c), which show $\chi_{1}^{W}$ for partial waves with $L=18$ and 12 . Angular momenta $L$ near
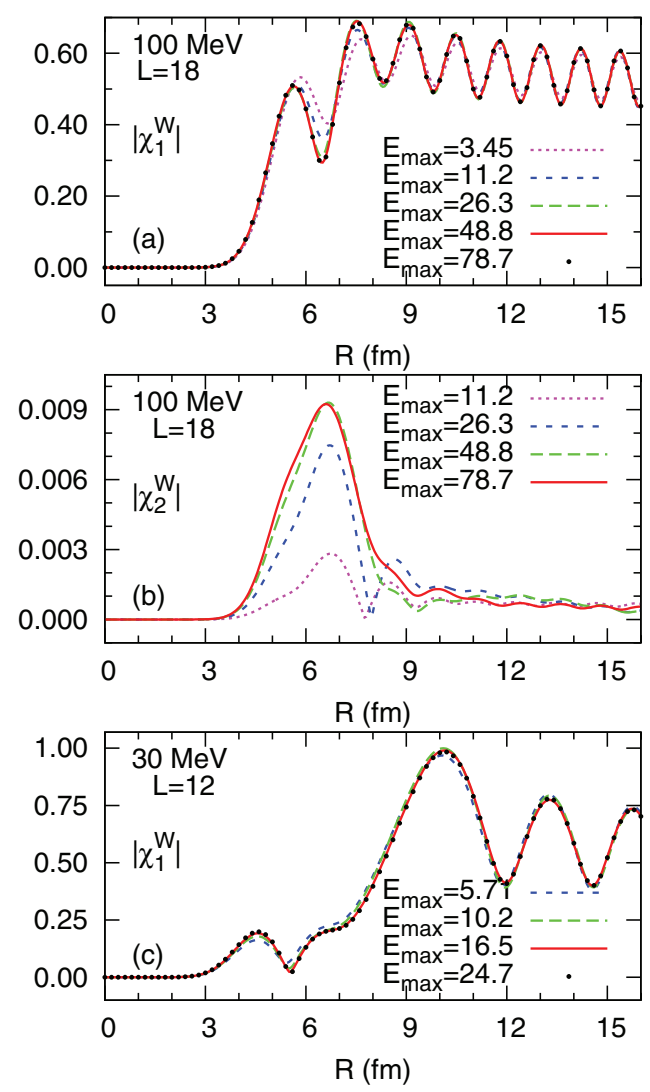

FIG. 2. (Color online) Convergence of selected partial waves of the Weinberg components $\chi_{i}^{W}$ with respect to the maximum $n$ - $p$ continuum energy included in Eq. (9). Results are for (a) $\chi_{1}^{W}$ and $E_{d}=100 \mathrm{MeV}$, (b) $\chi_{2}^{W}$ and $E_{d}=100 \mathrm{MeV}$, and (c) $\chi_{1}^{W}$ and $E_{d}=30$ $\mathrm{MeV}$. The partial wave values, $L$, associated with each $\chi_{i}^{W}$ are indicated in each panel.

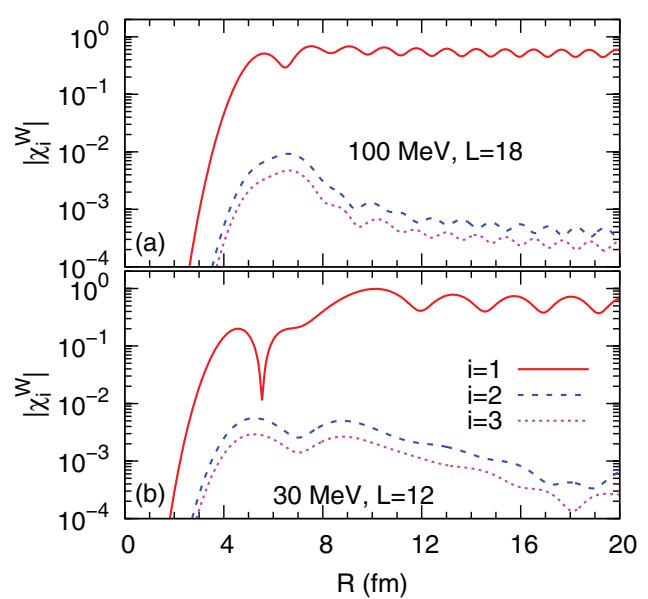

FIG. 3. (Color online) Calculated Weinberg state distorted waves $\chi_{i}^{W}$ demonstrating the dominance of $\chi_{1}^{W}$. Curves compare the moduli of $\chi_{1}^{W}, \chi_{2}^{W}$, and $\chi_{3}^{W}$ for the ${ }^{132} \operatorname{Sn}(d, p){ }^{133} \mathrm{Sn}$ reaction for (a) $E_{d}=$ $100 \mathrm{MeV}$ and partial wave $L=18$ and (b) $E_{d}=30 \mathrm{MeV}$ and partial wave $L=12$.

these values drive the dominant contributions to the $(d, p)$ reaction cross sections for $E_{d}=100$ and $30 \mathrm{MeV}$, respectively. Convergence of the $\chi_{i}^{W}$ with $i>1$ was not achieved, as anticipated from the behavior of the coefficients $C_{i j}$ shown in Fig. 1. We demonstrate this in Fig. 2(b) for $\chi_{2}^{W}$ and $E_{d}=100 \mathrm{MeV}$. As is expected, from the $C_{i j}$ dependence on $j$ for $i>1$, all $i>1$ Weinberg components are about two orders of magnitude smaller than $\chi_{1}^{W}$ in the most important radial region for the transfer amplitude. This is $R \approx 7 \mathrm{fm}$ in the present case (see Fig. 3).

The Weinberg distorted wave components $\chi_{i}^{W}$ constructed above contain contributions from all CDCC basis components within the range of $V_{n p}$. However, since $\chi_{1}^{W}$ dominates over all other Weinberg components, it is sufficient to perform onechannel transfer reaction calculations with only $\chi_{1}^{W}$ included. We call calculations truncated in this way DW $\chi_{1} \mathrm{~A}$ (distorted wave with $\chi_{1}^{W}$ approximation). For this purpose, we read the calculated $\chi_{i}^{W}(i=1,2,3)$ into the computer code TWOFNR [25] and calculate the transfer amplitude within the zero-range approximation. We use the same KD02 optical potential systematics as used in the deuteron channel for the proton distorted waves in the outgoing channel. In the model calculations presented, the neutron overlap function is approximated as a single particle wave function (with $\ell=3$ ) calculated using a Woods-Saxon potential with standard radius and diffuseness parameters, $r_{0}=1.25 \mathrm{fm}$ and $a_{0}=0.65 \mathrm{fm}$ and depth fitted to separation energy of $2.47 \mathrm{MeV}$ [26]. No spin-orbit potential was used for this wave function.

The $\mathrm{DW} \chi_{i} \mathrm{~A}$ differential cross sections are shown in Fig. 4 for 100 - and $30-\mathrm{MeV}$ incident deuteron energies, where the differential cross sections corresponding to each of $\chi_{1,2,3}^{W}$ and their coherent sum are shown. Evident from these figures is that the addition of channels $\chi_{2}^{W}$ and $\chi_{3}^{W}$ does not influence the cross sections at the forward angles where the angular distributions are usually measured and are most valuable for spectroscopy. The $\chi_{2}^{W}$ and $\chi_{3}^{W}$ contributions are noticeable at large angles where the cross sections are small, but even 


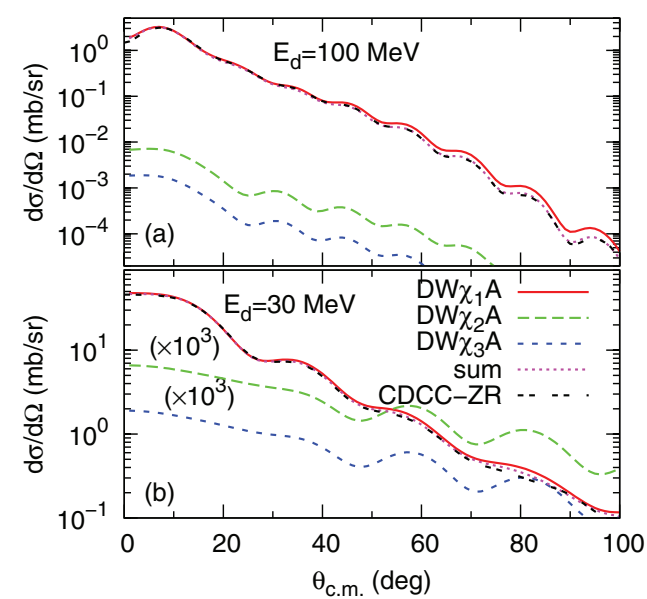

FIG. 4. (Color online) Comparisons of the calculated differential cross sections for the ${ }^{132} \mathrm{Sn}(d, p){ }^{133} \mathrm{Sn}$ reaction at (a) $100 \mathrm{MeV}$ and (b) $30 \mathrm{MeV}$, using Weinberg distorted wave components $\chi_{1}^{W}, \chi_{2}^{W}$, and $\chi_{3}^{W}$, showing the dominance of the first Weinberg component $\chi_{1}^{W}$; see the text for details.

there the changes are small. For comparison, the results of CDCC-ZR calculations, which include the contributions to transfer (in the zero-range approximation) from all of the CDCC continuum bins used to construct $\chi_{i}^{W}$, are also shown. As was expected, the cross sections from the CDCC-ZR calculation and from the coherent sums of the $\mathrm{DW} \chi_{i} \mathrm{~A}$ ( $i=1,2,3)$ amplitudes agree very well at both of the energies studied.

\section{SUMMARY}

Using as an example the ${ }^{132} \operatorname{Sn}(d, p){ }^{133} \mathrm{Sn}$ reaction at energies of 15 and $50 \mathrm{MeV} /$ nucleon typical of modern radioactive ion beam facilities, we have demonstrated that the dominant effects of deuteron breakup on calculations of $(d, p)$ reaction observables can be accommodated using a one-channel distorted-wave calculation. These calculations go well beyond the DWBA method in that no Born approximation step is involved. This calculation requires knowledge of an effective deuteron distorted wave, being the first component of the expansion of the $p+n+A$ scattering wave function $\Psi(\boldsymbol{r}, \boldsymbol{R})$ in Weinberg states. This component includes accurately breakup contributions from the small $n-p$ separations that dominate the $(d, p)$ reaction amplitude. It is defined as the projection of $\Psi(\boldsymbol{r}, \boldsymbol{R})$ onto the transfer reaction vertex, i.e., $V_{n p}\left|\phi_{d}\right\rangle$.

Johnson and Tandy [8] showed that, by neglecting couplings between components in the Weinberg expansion of the threebody wave function, one obtains a simple prescription for a potential that generates directly (an approximation to) the first Weinberg component. $(d, p)$ reaction calculations based on this approximation, known as the adiabatic distorted wave approximation (ADWA), have had some success in the analysis of data. Successful and more complete calculations that include the couplings between the Weinberg components have also been published [12]. We have shown here that there is a need to develop a simple procedure for correcting the ADWA, focusing specifically on calculating accurately only the first Weinberg component of the three-body scattering wave function $\Psi(\boldsymbol{r}, \boldsymbol{R})$. This would be especially important for incident energies of 3-10 MeV per nucleon, typical of TRIUMF [27], HRIBF at ORNL [28] (where the ${ }^{132} \mathrm{Sn}(d, p){ }^{133} \mathrm{Sn}$ reaction has been measured [29,30]), and ISOLDE [31], for which the influence of closed channels does not allow us to generate reliably this component using the scheme described above.

\section{ACKNOWLEDGMENTS}

DYP appreciates the warm hospitality he received during his visits to the University of Surrey. This work is supported by the National Natural Science Foundation of China under Grants No. 11275018, No. 11021504, and No. 11035001 and a project sponsored by the Scientific Research Foundation for Returned Overseas Chinese Scholars, State Education Ministry. NKT, RCJ, and JAT gratefully acknowledge the support of the United Kingdom Science and Technology Facilities Council (STFC) through research Grant No. ST/J000051.
[1] B. Jonson, Phys. Rep. 389, 1 (2004).

[2] W. N. Catford et al., Phys. Rev. Lett. 104, 192501 (2010).

[3] J. S. Thomas et al., Phys. Rev. C 76, 044302 (2007).

[4] A. M. Mukhamedzhanov, F. M. Nunes, and P. Mohr, Phys. Rev. C 77, 051601(R) (2008).

[5] R. C. Johnson and P. J. Soper, Phys. Rev. C 1, 976 (1970).

[6] J. D. Harvey and R. C. Johnson, Phys. Rev. C 3, 636 (1971).

[7] G. L. Wales and R. C. Johnson, Nucl. Phys. A 274, 168 (1976).

[8] R. C. Johnson and P. C. Tandy, Nucl. Phys. A 235, 56 (1974).

[9] J. Lee et al., Phys. Rev. Lett. 104, 112701 (2010).

[10] H. Amakawa, N. Austern, and C. M. Vincent, Phys. Rev. C 29, 699 (1984).

[11] E. J. Stephenson et al., Phys. Rev. C 42, 2562 (1990).

[12] A. Laid, J. A. Tostevin, and R. C. Johnson, Phys. Rev. C 48, 1307 (1993).

[13] A. Deltuva, A. M. Moro, E. Cravo, F. M. Nunes, and A. C. Fonseca, Phys. Rev. C 76, 064602 (2007).
[14] N. J. Upadhyay, A. Deltuva, and F. M. Nunes, Phys. Rev. C 85, 054621 (2012).

[15] N. K. Timofeyuk and R. C. Johnson, Phys. Rev. C 59, 1545 (1999).

[16] N. Austern, Y. Iseri, M. Kamimura, M. Kawai, G. Rawitscher, and M. Yahiro, Phys. Rep. 154, 125 (1987).

[17] I. J. Thompson, Comput. Phys. Rep. 7, 167 (1988).

[18] T. Kubo, M. Ishihara, N. Inabe, H. Kumagai, I. Tanihata, K. Yoshida, T. Nakamura, H. Okuno, S. Shimoura, and K. Asahi, Nucl. Instrum. Methods B 70, 309 (1992).

[19] A.C. C. Villari, Nucl. Instrum. Methods B 204, 31 (2003).

[20] D. J. Morrissey, Nucl. Phys. A 616, 45 (1997).

[21] A. M. Rodin et al., Nucl. Instrum. Methods A 391, 228 (2003).

[22] Z. Sun, W.-L. Zhan, Z.-Y. Guo, G. Xiao, and J.-X. Li, Nucl. Instrum. Methods A 503, 496 (2003).

[23] A. J. Koning and J. P. Delaroche, Nucl. Phys. A 713, 231 (2003). 
[24] J. A. Tostevin, F. M. Nunes, and I. J. Thompson, Phys. Rev. C 63, 024617 (2001).

[25] J. A. Tostevin, University of Surrey version of the code TWOFNR (of M. Toyama, M. Igarashi, and N. Kishida), http://www.nucleartheory.net/NPG/code.htm.

[26] G. Audi, A. H. Wapstra, and C. Thibault, Nucl. Phys. A 729, 337 (2003).
[27] P. G. Bricault, M. Dombsky, P. W. Schmor, and G. Stanford, Nucl. Instrum. Methods B 126, 231 (1997).

[28] J. R. Beene et al., J. Phys. G: Nucl. Part. Phys. 38, 024002 (2011).

[29] K. L. Jones et al., Nature (London) 465, 454 (2010).

[30] K. L. Jones et al., Phys. Rev. C 84, 034601 (2011).

[31] O. Kester et al., Nucl. Instrum. Methods B 204, 20 (2003). 